\title{
Identification of surface features on cold-rolled stainless steel strip
}

\author{
R. Ahmed ${ }^{1}$, M.P.F. Sutcliffe* \\ Cambridge University, Department of Engineering, Trumpington Street, Cambridge, CB2 IPZ, UK \\ Received 9 July 1999; received in revised form 17 May 2000; accepted 15 June 2000
}

\begin{abstract}
A novel method based on three-dimensional profilometry data and MATLAB analysis software is described to identify surface features on cold-rolled stainless steel strip. The aim of the method is to detect automatically pits and roll marks that can be observed in optical or SEM micrographs. Pits are identified by locating regions which are significantly deeper than the immediately adjacent surface. Deep or steep features which extend a significant distance in the direction of rolling are identified as roll marks. Results for typical cold-rolled stainless steel sheet show that the algorithms are effective in identifying the more obvious pits and roll marks. By suitable adjustment of the tolerances used in the analysis, the method can be tailored to detect less severe features. Application of the method, either for research purposes or routine industrial inspection, will require tuning of these tolerances to detect pits of the severity relevant to the end use of the strip. The methodology has been applied to a series of rolled strip samples to track the evolution of pits and roll marks during a schedule. Results show how the initially large area of deep pits is rapidly eliminated and transformed into shallow pits. The pit identification method is used to estimate the effect of trapped oil on lubrication. Results suggest that this expelled oil will contribute significantly to the lubrication of the surrounding area. Finally, a good correlation is demonstrated between strip surface reflectance measurements and the estimated pit area. () 2000 Elsevier Science S.A. All rights reserved.
\end{abstract}

Keywords: Stainless steel; Rolling; Pits; Voids; Surface characterisation

\section{Introduction}

Control of surface finish is a major concern in the manufacture of stainless steel strip. Prior to the cold-rolling operation, the hot band is annealed, shot-blast and pickled, leaving a comparatively rough and broken surface, as illustrated in the scanning electron micrograph of Fig. 1a. The bright finish or gloss required for many products is then generated during the cold-rolling operation on a cluster Sendzimir mill. The flattening of the strip asperities during rolling, particularly with a smooth roll surface, is greatly enhanced by the bulk deformation of the underlying material $[1,2]$. As the strip is deformed, the deep features of the pickled surface are eliminated, to leave a large number of relatively shallow pits, as illustrated in Fig. 1b and c. These pits can significantly affect the visual appearance of the strip, and may also play an important role in trapping lu-

\footnotetext{
* Corresponding author. Present address: Department of Mechanical and Chemical Engineering, Heriot-Watt University, Riccarton, Edinburgh, EH14 4AS, UK.

E-mail address: mpfs@eng.cam.ac.uk (M.P.F. Sutcliffe).

${ }^{1}$ Present address: Department of Mechanical and Chemical Engineering, Heriot-Watt University, Riccarton, Edinburgh, EH14 4AS, UK.
}

bricant during subsequent sheet forming operations. During rolling, roll grinding marks can also be transferred to the strip to leave a surface finish on the strip, as illustrated in the optical micrograph Fig. 4a, which may be undesirable. Hydrodynamic entrainment of oil into the bite tends to keep the surfaces separated and prevents effective flattening of the asperities on the strip [3-5]. However, it also seems likely that a significant amount of lubricant is trapped in isolated pits on the strip surface. Build-up of hydrostatic pressure in this trapped oil will tend to prevent these features being eliminated. Experimental measurements by Mizuno and Okamoto [6] with an artificial rough work-piece surface show how trapped oil can be drawn out of the pits during the rolling process by the sliding action between the strip and the roll. An alternative approach considers the way in which oil is not completely trapped in the pits, but can flow out along channels in the rolling direction [7,8]. Various experimental [9-11] and analytical [12] studies have considered these mechanisms, but the details of the lubrication mechanisms and the evolution of surface features remains unclear.

Previous studies to quantify the evolution of surface features during cold rolling have been limited. Gjønnes [13] used laser-scanning microscopy to identify grooves, roll 


\begin{tabular}{|c|c|}
\hline \multicolumn{2}{|c|}{ Nomenclature } \\
\hline$d$ & Pit depth matrix \\
\hline$h$ & Surface height matrix \\
\hline$i, j$ & $\begin{array}{l}\text { Indices in the rolling and transverse direction, } \\
\text { respectively }\end{array}$ \\
\hline$n$ & $\begin{array}{l}\text { Number of points averaged to establish a } \\
\text { mean height }\end{array}$ \\
\hline$r$ & Preliminary identification matrix for a roll mark \\
\hline$R_{\mathrm{qr}}$ & Roll r.m.s. roughness amplitude \\
\hline$L$ & $\begin{array}{l}\text { Spanning length for estimating the mean height } \\
\text { either side of a pit }\end{array}$ \\
\hline$M$ & $\begin{array}{l}\text { Length scale in the rolling direction for } \\
\text { identifying roll marks }\end{array}$ \\
\hline \multicolumn{2}{|c|}{ Greek letters } \\
\hline$\delta_{\mathrm{m}}$ & $\begin{array}{l}\text { Depth tolerance parameter for roll mark } \\
\text { identification }\end{array}$ \\
\hline$\delta_{\mathrm{p}}$ & Depth tolerance parameter for pit identification \\
\hline$\Delta$ & Sampling distance of surface measurements \\
\hline$\phi$ & Slope tolerance parameter \\
\hline & $\begin{array}{l}\text { Matrix of surface slopes estimated numerically } \\
\text { from height data }\end{array}$ \\
\hline
\end{tabular}

ridges and 'shingles' on cold-rolled aluminium. Ohkubo et al. [14] investigated the influence on the surface gloss of aluminium foil of oil pits, 'slags', roll marks and small depressions. They quantified these defects using two-dimensional scanning electron microscope and three-dimensional atomic force microscope observations, concluding that the dominant factor in determining surface gloss was oil pits. Kuznetsov [15] used a theoretical approach to derive a work-roll imprint factor, assessing the influence of work-roll wear on the formation of roll marks on the strip surface. Most of these studies relate to aluminium rolling. However, rolling of stainless steel strip differs significantly from aluminium rolling as the pickling and shot blasting processes used in steel rolling may introduce gross surface defects which then have to be removed.

Current industrial practice in stainless steel rolling is to use surface reflectance and 'distinctness of image' tests to characterise the surface quality of rolled sheet. However, these techniques are sensitive to the surface chemistry, sheet roughness and angle of incidence, making accurate quantitative comparisons difficult. Moreover, such approaches provide no indication of the potential origin of poor surface quality. There is, therefore, an urgent need for alternative methods to be developed in order to better characterise the sheet surface quality. Recent advancements in surface profilometry (higher resolution, larger measurement areas and shorter measurement times) have made this possible. For example, Schmoeckel and Staeves [16] apply a functional filter to three-dimensional profilometry data to assess the tribological performance of steel sheet. Recent work by Le and Sutcliffe [17] describes a Fourier transform filtering
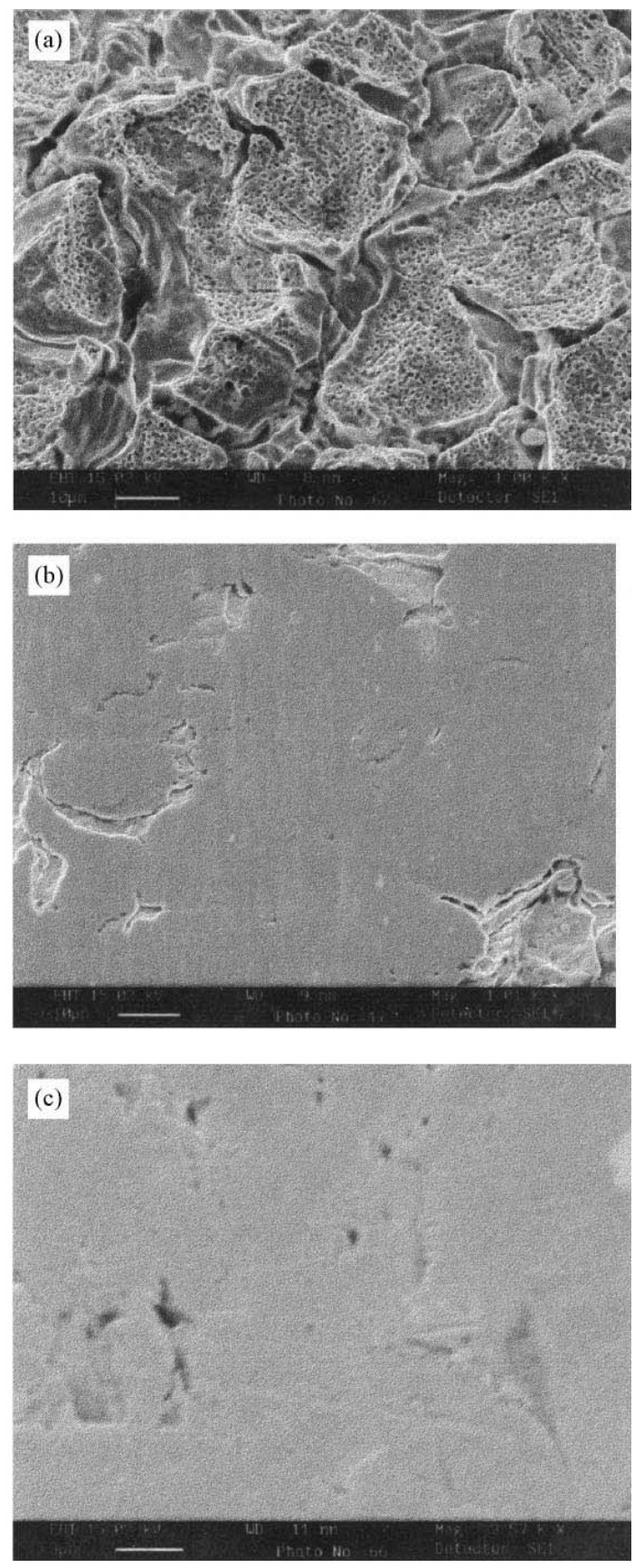

Fig. 1. SEM micrographs of the sheet surface after various passes: (a) the shot-blast and pickled surface; (b) after an intermediate pass; and (c) after the final pass.

technique to estimate the pit area on rolled aluminium sheet while Jiang et al. [18] apply wavelet analysis to surface roughness characterisation.

The aim of this paper is to use three dimensional profilometry to characterise the sheet quality based upon the detection of surface features. The methodology used to identify pertinent features is presented in detail, and illustrated by examining the evolution of these surface features during a typical 
pass schedule. Results are interpreted in terms of lubrication mechanisms and correlated with surface reflectance tests.

\section{Experimental test procedure}

Samples of austenitic stainless steel sheet, of width $1.3 \mathrm{~m}$ and initial thickness $4.0 \mathrm{~mm}$, were collected at various intermediate stages of the manufacturing operation. The hot band was annealed, shot-blasted and pickled prior to being cold rolled in a 20-high Sendzimir mill to a final thickness of $1.5 \mathrm{~mm}$. The work rolls, which were $\approx 50 \mathrm{~mm}$ in diameter, were ground after the first pass and then replaced with polished rolls towards the end of the schedule. A low-viscosity rolling oil with a kinematic viscosity $<10 \mathrm{cSt}$ at $40^{\circ} \mathrm{C}$ was used. Strip samples were collected from across the width of the strip at either end of the coil after selected passes. As the samples were collected from positions on the coil after mill stoppage, potential effects due to hydrodynamic lubrication (arising from the entraining action at normal rolling speeds) will not be present.

\section{Surface profilometry}

A Zygo three-dimensional white-light interferometeric profilometer was used to measure the surface roughness of the sheet samples. The instrument has maximum lateral and vertical resolutions of $\approx 0.5 \mu \mathrm{m}$ and $0.1 \mathrm{~nm}$, respectively. Typically, the measurement area was $0.34 \mathrm{~mm} \times 0.26 \mathrm{~mm}$. The data was recorded over an array of $320 \times 240$ pixels and the pixel spacing for the standard measurements was about $1.1 \mu \mathrm{m}$. For the initial shot-blast surface, a larger measurement area of $2.75 \mathrm{~mm} \times 2.06 \mathrm{~mm}$ was used, with a correspondingly larger pixel spacing. The surface roughness of the work rolls was measured by replicating the roll surface using a 'press-on-film' supplied by Testex, consisting of a layer of crushable plastic microfoam on a polyester substrate. The film was placed on the roll surface and rubbed using a burnishing tool to transfer the roll topography to the film. The accuracy of the technique was verified by comparing the roughness measurements of a sheet surface and its corresponding replica. Measurements of the strip and roll surface for the various samples were repeated on three regions of each strip. No filtering of the profile data was used, except for subtraction of the mean plane. The initial roll roughness r.m.s. amplitude varied from $0.15 \mu \mathrm{m}$ for the earlier passes to $0.05 \mu \mathrm{m}$ towards the end of the schedule. The roughness of the rougher rolls decreased slightly during the pass schedule while the smoother rolls did not change significantly. The in-going strip had an r.m.s. roughness amplitude of $6.9 \mu \mathrm{m}$, which was reduced to about $0.06 \mu \mathrm{m}$ after the final pass. Identification of surface features, as described in the following section, was carried out by importing surface height data from the profilometry measurements into the mathematical package MATLAB for subsequent processing.

\section{Observation of surface features}

Fig. 1a shows a scanning electron microscope (SEM) image of the in-going sheet after annealing, shot blasting and pickling. Various features can be observed, including shot-blast craters, fissures at the grain boundaries caused by preferential etching and micro-pitting within the grains. Fig. $1 b$ and $c$ show SEM images of the strip surface after an intermediate and the final passes, respectively, while Fig. 3a and Fig. 4a show the corresponding optical micrographs. For all these figures, the rolling direction runs in the vertical direction. Pits are typically $5-10 \mu \mathrm{m}$ in size. Although the size and frequency of the larger surface pits decrease during the rolling schedule, a significant number of small features persist after the final pass. The optical micrographs show clearly the way that the longitudinal roughness of the rolls, generated during roll grinding, is transferred to the strip surface as the strip conforms to the roll roughness in the bite.

\section{Identification of surface features - methodology}

The above section shows that the main features observed on these strip surface samples are pits and roll marks. Algorithms described in this section aim at identifying these features automatically, to allow a quick, accurate and objective quantification of the relative proportions of these features.

\subsection{Identification of pits}

A large number of dark, rather irregular pits can be observed on both the optical and SEM micrographs of Figs. 1, 3 and 4. Observations, both from such micrographs and from profilometry data, show that the size and depth of these pits changes during the rolling schedule, but that they are mainly characterised by having rather steep sides, so that the surface of a pit lies significantly below the adjacent surface. This is illustrated by a typical two-dimensional trace taken along the rolling direction (Fig. 2). Algorithms are described below to identify such pits, based on this observation.

Consider a matrix containing surface height data obtained by three-dimensional profilometry. Elements of the matrix are denoted by $h(i, j)$, where changes in the $i$ th index represent variations in the rolling direction. To establish the mean surface height on either side of the current data point, we take the mean of $n$ points at around a distance $L$ away from the point under consideration. Taking a profile transverse to the rolling direction, the pit depth $d_{\mathrm{t}}(i, j)$ relative to the surrounding area is thus given by 


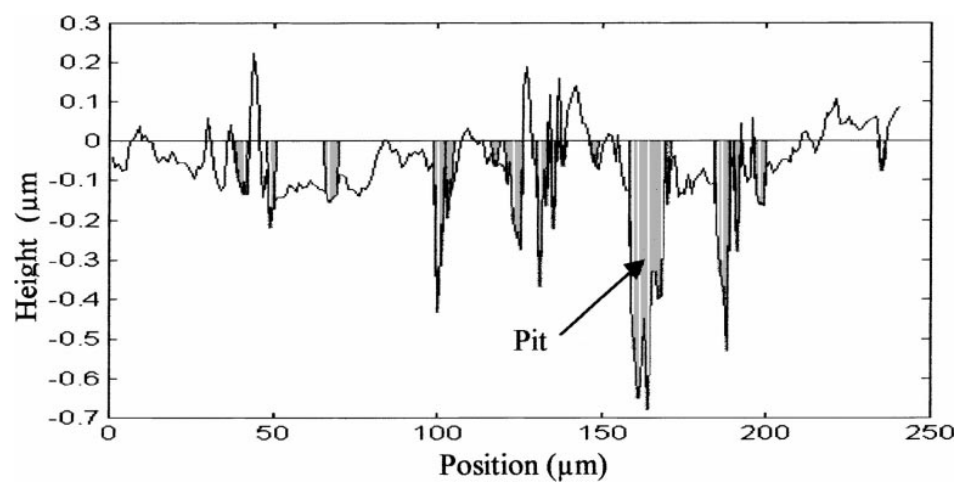

Fig. 2. Typical profile of strip after an intermediate pass taken in the rolling direction. Pits identified using $\delta_{\mathrm{p}}=0.66$ and $L=27 \mu \mathrm{m}$ are shown shaded.

$d_{\mathrm{t}}(i, j)=\frac{1}{2 n}\left(\sum_{k=j-L / \Delta}^{k=j-L / \Delta+n-1} h(i, k)+\sum_{k=j+L / \Delta-n+1}^{k=j+L / \Delta} h(i, k)\right)-h(i, j)$

Here, $\Delta$ is the pixel spacing of the profilometer data (see Section 3). The spanning length $L$ should be chosen to be greater than the width of a typical pit, with $L / \Delta$ an integer. A value of $n=4$ was found to be appropriate to estimate the height of the flat regions around the pit. A similar expression is used to estimate the pit depth $d_{\mathrm{r}}(i, j)$ based on a profile in the rolling direction, with the summation of Eq. (1) now carried out over the $i$ th index. The same values of the parameters $L$ and $n$ are used for this longitudinal direction. A given data point is identified as being in a pit when the depth of the pit is greater than a critical value. Because of the relatively thin oil films in the rolling operation, we expect the unpitted region to have an r.m.s. roughness $R_{\mathrm{q}}$ close to that of the rolls. Hence, it is appropriate to scale the pit depth criterion by the roll roughness $R_{\mathrm{qr}}$, so that the criterion for identifying a pit becomes

$$
\frac{d_{\mathrm{r}}(i, j)}{R_{\mathrm{qr}}}>\delta_{\mathrm{p}} \text { and } \frac{d_{\mathrm{t}}(i, j)}{R_{\mathrm{qr}}}>\delta_{\mathrm{p}}
$$

where $\delta_{\mathrm{p}}$ is a dimensionless tolerance for the pit depth criterion, typically of the order one. In applying this tolerance, although significantly smoother rolls were used for the final passes, nevertheless the roughness of the rolls used for the earlier passes (i.e. $0.15 \mu \mathrm{m}$ ) was used throughout. The effect of this is discussed in Section 6.2. To allow for the possibility of a neighbouring pit influencing the results of the above analysis, the procedure described was refined by repeating the analysis of equations (1) and (2), replacing $L$ by $L / 5$ and taking a value of $n=2$. Any points satisfying the criterion of Eq. (2) with either $L$ and $n=4$ or $L / 5$ and $n=2$ were included as pitted regions.

Having identified which pixels in an image are pits, the total pit area, expressed as a proportion of the total area of the sample, can be straightforwardly evaluated, as can the 'deep-pit area', for a pit depth below $0.5 \mu \mathrm{m}$.

It is evident that it will be difficult to identify small pits whose depth is of the order of the roll roughness. The above method, using rather small values of $n$ to identify a mean height, will suffer from inaccuracies in establishing a mean height. However, the obvious solution of taking more points to establish a mean height for the unpitted area does not necessarily lead to an increase in accuracy, as it becomes increasingly likely that the set of points used to find the plateau height now includes an adjacent pit. The above method was chosen as a compromise. The validity of the approach is justified and appropriate values for the tolerance parameters suggested in Section 6.1, where results are compared with visual observations of the pits.

\subsection{Identification of roll marks}

Section 4 shows how, as the strip is reduced in thickness, grind marks on the roll may be transferred to the strip. These roll marks are characterised by being long, straight valleys or peaks running in the direction of rolling. Although the roll surface has a distribution of asperity shapes, it is only those roll marks generated on the strip surface which have a large height, depth or slope which will significantly affect its visual appearance. Algorithms are described below which identify and quantify these roll marks, based on these observation.

The method described in Section 5.1 is used to assess the amplitude of the roll mark relative to the surrounding area at a distance $L$ from the point under consideration, with the asperity depth $d_{\mathrm{t}}(i, j)$ given by Eq. (2). For these relatively thin features, the number of points $n$ averaged to find the height of the adjacent region was taken as 2 . The slope $\theta(i, j)$, taking a profile in the transverse direction, is given by

$\theta(i, j)=\frac{h(i, j)-h(i, j+1)}{\Delta}$

A preliminary identification of roll marks is made by comparing the magnitude of the strip asperity depth and slope with an appropriate tolerance value. Hence, a preliminary 
identification matrix $r(i, j)$ is given a value of one, representing a putative roll mark, when

$\left|\frac{d_{\mathrm{t}}(i, j)}{R_{\mathrm{qr}}}\right|>\delta_{\mathrm{m}} \quad$ or $\quad\left|\frac{\theta(i, j)}{R_{\mathrm{qr}} / \lambda_{\mathrm{r}}}\right|>\phi$

$r(i, j)$ is put equal to zero when the above criterion is false. The dimensionless tolerances, $\delta_{\mathrm{m}}$, for the roll mark depth and $\phi$ for the slope, are of the order of one. As with the pit analysis, for all passes the parameters for the roll used in the initial and intermediate passes are taken. This has the effect required in practice of only picking up the severe roll marks coming from these rougher rolls, as discussed in Section 6.2. This roll has an roughness amplitude $R_{\mathrm{qr}}$ of $0.15 \mu \mathrm{m}$ and a length scale $\lambda_{\mathrm{r}}$ of $15 \mu \mathrm{m}$ (taking the distance at which the autocorrelation function falls to a value of 0.1 ), giving a typical slope $R_{\mathrm{qr}} / \lambda_{\mathrm{r}}$. of 0.01 . Roll marks are characterised by extending significantly in the rolling direction. To verify this, the preliminary identification matrix $r(i, j)$ is scanned to pick out any features which extend a distance approximately equal to $M$ in the rolling direction, giving a final criterion for identifying roll marks as

$\sum_{i=-M / \Delta}^{i=M / \Delta} r(i, j) \geq \frac{M}{\Delta}$

where $M$ should be chosen so that $M / \Delta$ is an integer. In practice it was found helpful to put equal to zero the height $h(i, j)$ obtained from the three-dimensional profilometry for those pixels identified as pits, before using the above algorithm.

\section{Identification of surface features - results}

The methodology of feature identification described above is applied in Section 6.1 to typical strip samples. Emphasis is placed on relating visual observations to the features identified using the new algorithms. Section 6.2 applies the method to a series of passes to show how features evolve during the pass schedule. In Section 6.3, a sensitivity analysis is performed, to give guidance on an appropriate choice of the various parameters needed in the identification algorithm.

\subsection{Application of the methodology to typical strip surfaces}

In this section, the methodology of Section 5 is applied to strip samples taken after an intermediate and the final pass. Fig. 3a shows an optical micrograph of the sheet surface after an intermediate pass and Fig. $3 b$ gives the corresponding surface map obtained from the profilometer, with the grey-scale intensity representing height according to the scale on the figure. The same part of the surface is covered in both Fig. $3 a$ and b, with an area of view of $340 \mu \mathrm{m} \times 260 \mu \mathrm{m}$. For this pass, the rougher $0.15 \mu \mathrm{m}$ rolls were used. The dark $\operatorname{areas}^{2}$ shown on Fig. 3c mark the location of pits identified using the methodology of Section 5.1 with a pit depth tolerance $\delta_{\mathrm{p}}=0.33$ (based on $R_{\mathrm{qr}}=0.15 \mu \mathrm{m}$ ) and pit spanning length $L=27 \mu \mathrm{m}$. Fig. $3 \mathrm{~d}$ shows the relatively small number of pits deeper than $0.5 \mu \mathrm{m}$, identified using the same parameters. Fig. 3e shows the effect on the estimate of the pit area of taking a larger pit depth tolerance $\delta_{\mathrm{p}}=0.66$. A comparison of the visual plots, Fig. $3 \mathrm{a}$ and $\mathrm{b}$ with the analysis plots, shows that the pits observed on the strip surface are clearly identified using the larger depth pit tolerance $\delta_{\mathrm{p}}=0.66$, Fig. $3 \mathrm{e}$, confirming the effectiveness of the algorithm. With the smaller pit tolerance $\delta_{\mathrm{p}}=0.33$, Fig. $3 \mathrm{c}$, many smaller pits are identified, although it is likely that in this case the accuracy in identifying pits is reduced and some of these features are artefacts of the identification method. The location of pits identified using $\delta_{\mathrm{p}}=0.66$ and $L=27 \mu \mathrm{m}$ are marked by the shaded regions on Fig. 2, which shows a typical profilometer trace taken along the rolling direction. This figure confirms that the deeper pits are clearly identified. The results for the smaller pits are less conclusive. In these cases, it is easier to judge the accuracy of the estimates by comparing the analysis with the optical and profilometry data (Fig. 3). The value of spanning length $L$ is selected as being slightly larger than the biggest pits observed.

An appropriate choice of pit tolerance depends on what purpose the pit quantification is to be used for. In many cases, only the larger pits identified using $\delta_{\mathrm{p}}=0.66$ may be relevant. If even the smaller pits are important, the loss of accuracy for a value of $\delta_{\mathrm{p}}=0.33$ in identifying the pit area may be acceptable. The sensitivity to these choices is further explored in the next section.

Similar results are shown in Fig. 4 for the final pass, which was rolled with the smoother rolls of roughness $0.05 \mu \mathrm{m}$. An optical micrograph and corresponding surface map for this pass are given in Fig. 4a and b, while Fig. 4c shows the total pit area identified using $L=27 \mu \mathrm{m}$ and $\delta_{\mathrm{p}}=0.33$ (based on $R_{\mathrm{qr}}=0.15 \mu \mathrm{m}$ ). Pits seen on the visual plots are clearly observed using the identification algorithm. The depth tolerance parameter $\delta_{\mathrm{p}}$ has been based on the roughness of the rougher rolls used for the earlier passes, so as to be consistent with the results presented in the following section. A comparison of Fig. 3c and Fig. 4c, with the same nominal value of $\delta_{\mathrm{p}}=0.33$, shows that the accuracy of the analysis is much greater for the final pass. In this case, a better indication of the reliability of the identification method would have been obtained by basing $\delta_{\mathrm{p}}$ on the actual roll roughness for this pass to give a value of $\delta_{\mathrm{p}}=1.0$.

Turning to the identification of roll marks, Fig. $3 \mathrm{f}$ shows results on the strip sample taken after an intermediate pass, with a roll mark depth tolerance $\delta_{\mathrm{m}}=0.66$, slope tolerance $\phi=10$, spanning length $L=16 \mu \mathrm{m}$ and roll mark length $M=11 \mu \mathrm{m}$. Dark areas on this figure correspond to roll marks below the mean surface, while light areas are roll

\footnotetext{
${ }^{2}$ Note that the grid of small black dots on this figure (and similar dark or light dots on other figures) are an artefact of the plotting routine used.
} 

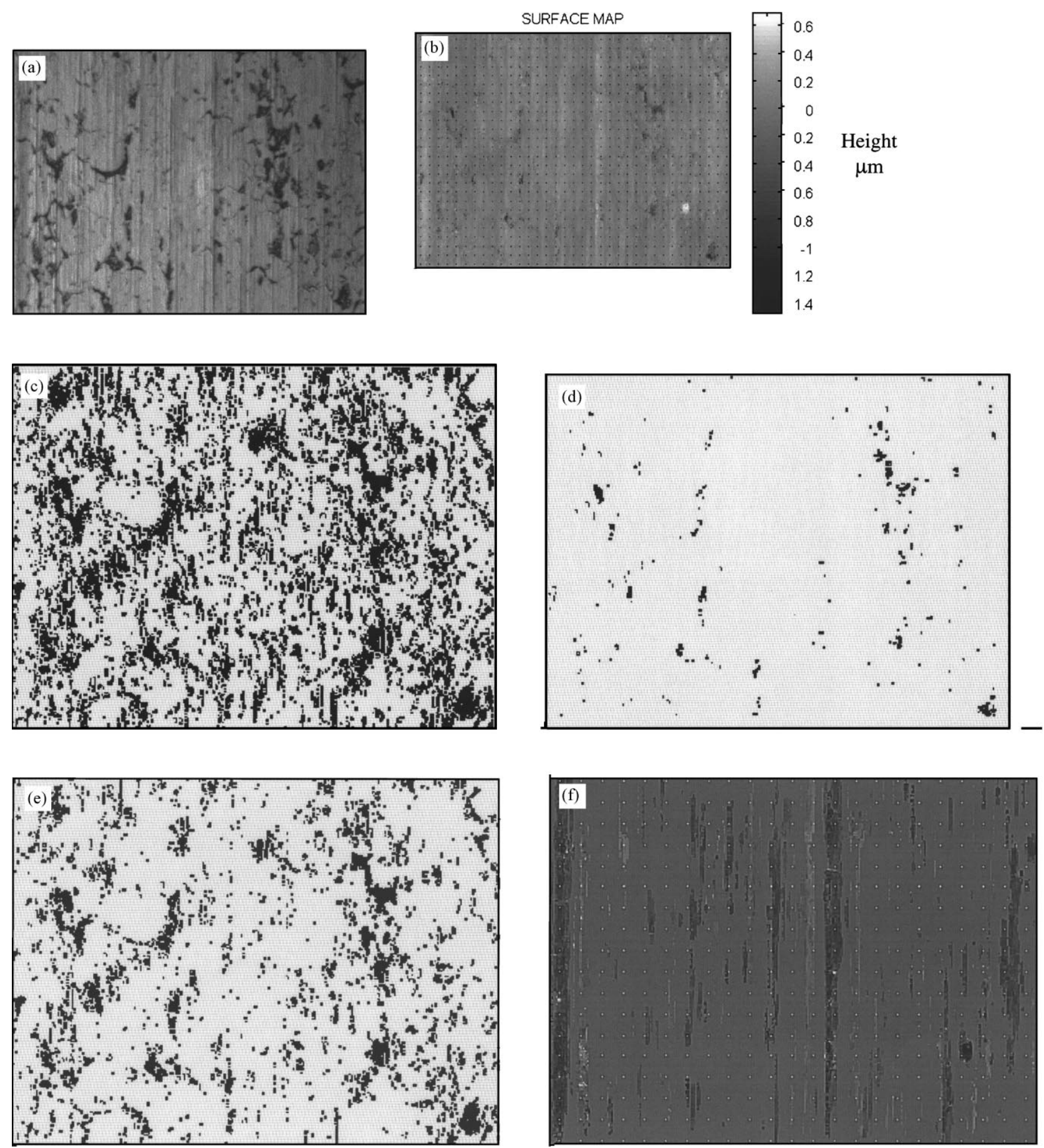

Fig. 3. Surface features on a strip sample taken after an intermediate pass. The measurement area is $340 \mu \mathrm{m} \times 260 \mu \mathrm{m}$ in all cases: (a) optical micrograph; and (b) corresponding surface map obtained from profilometry data. The grey-scale indicates the surface height: (c) pits identified using $\delta_{\mathrm{p}}=0.33$ and $L=27 \mu \mathrm{m}$; (d) pits deeper than $0.5 \mu \mathrm{m}$ identified using $\delta_{\mathrm{p}}=0.33$ and $L=27 \mu \mathrm{m}$; (e) pits identified using $\delta_{\mathrm{p}}=0.66$ and $L=27 \mu \mathrm{m}$; and (f) roll-grind marks identified using $\delta_{\mathrm{m}}=0.66, \phi=10, L=27 \mu \mathrm{m}$ and $M=11 \mu \mathrm{m}$.

marks above the mean surface. A comparison of this figure with the optical view, Fig. 3a, shows that most work roll marks have been identified. As with the pit identification, smaller values for the various tolerances can be used to detect less obvious roll marks. Fig. 5 shows similar results for a replica taken from a freshly ground roll used during the intermediate passes, using the same algorithm tolerances as above. Again dark areas correspond to valleys on the replica, and hence to peaks on the roll surface. A comparison of Fig. $3 \mathrm{f}$ for the strip after an intermediate pass and 

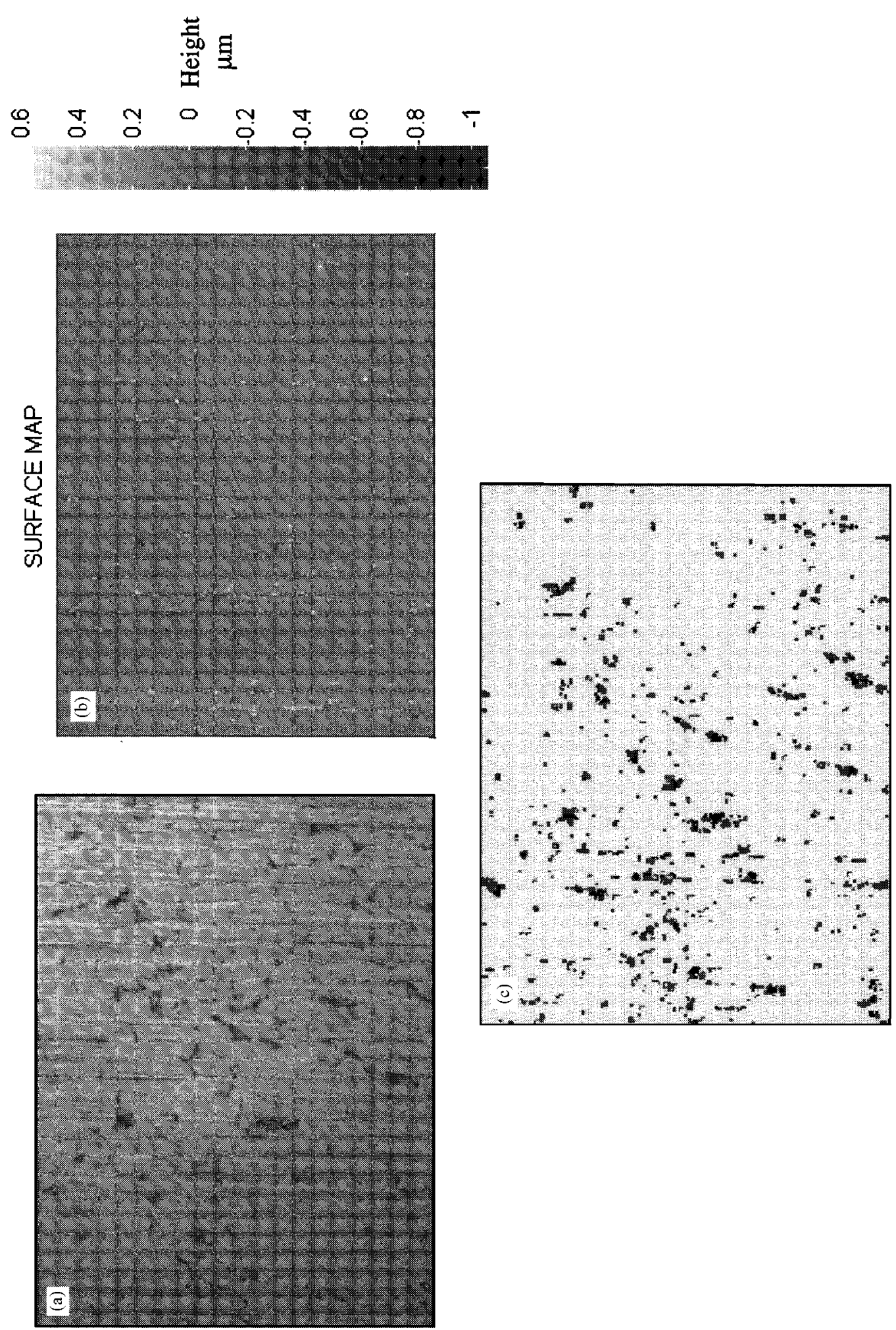


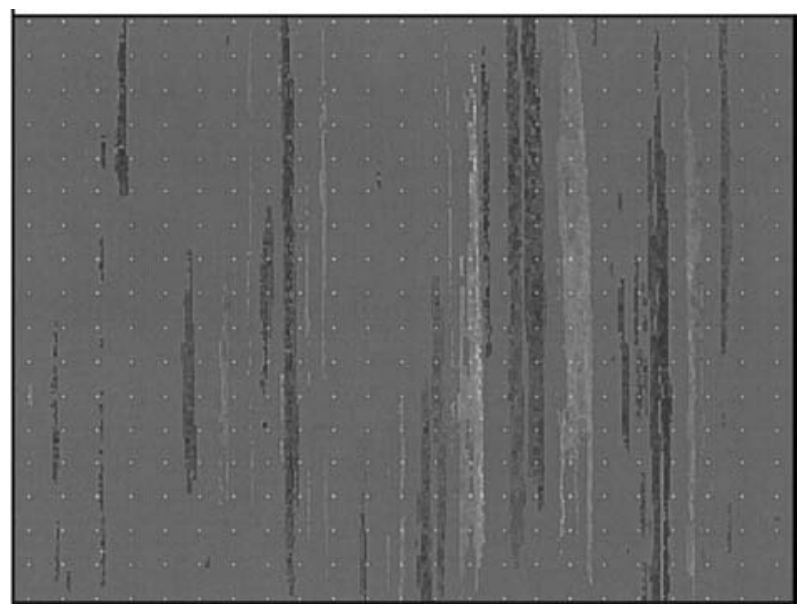

Fig. 5. Roll-grind marks identified on a replica of a freshly ground roll using $\delta_{\mathrm{m}}=0.66, \phi=10, L=27 \mu \mathrm{m}$ and $M=11 \mu \mathrm{m}$. The measurement area is $340 \mu \mathrm{m} \times 260 \mu \mathrm{m}$.

Fig. 5 for the roll replica show that the pattern of grind marks on the work roll is very similar to that for the roll marks seen on the strip, confirming the origin of the roll marks. The relative area of grinding marks on the roll is $\approx 19 \%$, similar to the roll mark area of $21 \%$ on the sheet surface for the intermediate pass.

\subsection{Evolution of surface features during the pass schedule}

The methodology described in Section 5 is applied to samples collected during the pass schedule, as described in Section 2, to track the evolution of surface features. To explore the effect of pit tolerance parameter on the pit analysis, values of $\delta_{\mathrm{p}}=0.33$ or 0.60 are used throughout the pass schedule, with $L=27 \mu \mathrm{m}$. For the roll marks, values of $\delta_{\mathrm{m}}=0.66$, $\phi=10, L=27 \mu \mathrm{m}$ and $M=11 \mu \mathrm{m}$ are used. Fig. 6 shows the evolution of total pit area, deep pit area (for pits deeper than

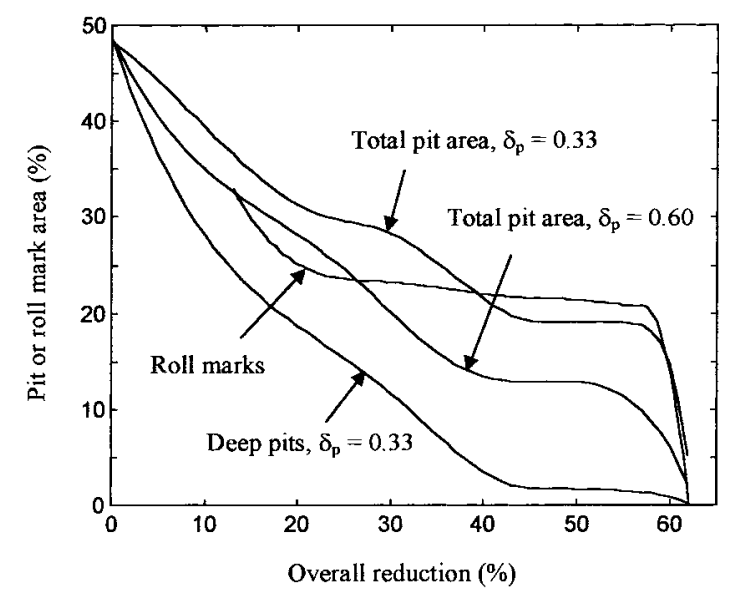

Fig. 6. Evolution of the relative area of surface features during the pass schedule.
$0.5 \mu \mathrm{m})$ and roll mark area during the pass schedule as a function of the overall reduction in strip thickness. It is worth remembering that the samples collected were taken from the ends of the coil (at reduced speed), so that these results may not be typical of strip rolled under normal conditions. Fig. 6 shows how the initially large area of deep pits, due to shot-blast damage and grain boundary etching, is rapidly reduced. After several passes most of these deep pits have been eliminated or transformed into shallower pits. In the final passes, the area of the shallower pits is further reduced.

Results are qualitatively similar for both values of the depth parameter $\delta_{\mathrm{p}}$ although the actual pit area is larger for the smaller value of $\delta_{\mathrm{p}}$. Some of this difference in the later passes may be due to inaccuracies for $\delta_{\mathrm{p}}=0.33$. Probably the value of total pit area with $\delta_{\mathrm{p}}=0.60$ gives a more appropriate measure of the relevant pits (cf. the analyses of Fig. 3). The depth tolerance parameter has been defined for all passes using the $\mathrm{R}_{\mathrm{q}}$ of the rougher rolls used for the earlier passes. By using the same value of $\delta_{\mathrm{p}}$ throughout the pass schedule, this means that pits of the same absolute depth are identified. Since pit identification will be more accurate in the later passes where the smooth rolls are used, this would lead to a reduction in the estimated pit area at the change to the smoother rolls, irrespective of the actual changes. However, the results of Fig. 3 suggest that this effect is relatively insignificant using $\delta_{\mathrm{p}}=0.60$. The alternative strategy of retaining the same value of $\delta_{\mathrm{p}}$, but now based on the true roll roughness which changes through the pass schedule, would introduce its own difficulties, as the absolute value of the depth tolerance would now change significantly between passes.

The roll mark area stays relatively constant up to the final pass, as the strip conforms to the roll during bulk deformation. In the final pass, the strip now conforms to the much smoother roll surface and most of the roll marks left on the surface from previous passes are eliminated. For the sample taken after the final pass, it is useful to identify any grinding marks left from the intermediate passes which are not eliminated in the final pass. This is precisely the effect achieved in Fig. 6, using tolerance parameters based on the roughness of the roll used for the first passes, as described in Section 5.2. Although we expect that any grinding marks on the smooth roll used in the final pass would be transferred to the strip, these small amplitude marks would not significantly affect the strip visual appearance.

\subsection{Sensitivity analysis}

Section 6.1 has demonstrated the applicability of the pit and roll mark identification methods. In this section, the sensitivity of results to the various parameters required is investigated. By presenting results for the pit area and roll mark as a proportion of the sample area, results should not be sensitive to the sample area as long as the measurement area is sufficiently large to be representative. A comparison of results for the area used, $340 \mu \mathrm{m} \times 260 \mu \mathrm{m}$, with measure- 
ments at different magnifications confirmed that this size of area is adequate. Estimates of percentage pit area using measurements at three locations on the surface of a sample from one of the earlier passes typically varied by a maximum of $\pm 3 \%$, for a mean pit area of about $30 \%$. Optical micrographs confirm that the pixel spacing of $1.1 \mu \mathrm{m}$ at the magnification used is adequate to resolve most of the relevant features on the surface. Although the geometry of the smaller pits will not be accurately defined, this is not considered to be a significant source of error.

In order to identify a mean height of the surrounding area, values of $n=2$ and 4 have been used for the pit-and-roll mark identifications, Eq. (1). These small values were chosen to avoid errors in estimating the plateau height by including adjacent pits in the calculation. With these rather small values, the mean height will not be accurate enough to give reliable estimates of pit area for shallow pits associated with small values of the tolerance parameters $\delta_{\mathrm{m}}$ and $\delta_{\mathrm{p}}$. The results, Figs. 3-5, suggest that, for a value of $d$ equal to 0.66 , this source of error does not lead to significant errors in locating the pits and roll marks. For a value of $\delta_{\mathrm{p}}=0.33$, Fig. $3 \mathrm{c}$, it seems likely that some of the smaller pits are erroneously identified or are not detectable in the optical micrograph.

\subsubsection{Pits}

The pit identification procedure contains two parameters, the pit depth tolerance $\delta_{\mathrm{p}}$ and pit spanning length $L$. Two approaches are taken to assess an appropriate choice of these parameters, by comparison with observations and by a sensitivity analysis. Section 6.1 illustrates how the choice of pit-depth tolerance determines the number of pits detected, with a tolerance $\delta_{\mathrm{p}}=0.66$ appropriate to identify only the more prominent pits, while $\delta_{\mathrm{p}}=0.33$ could be used to include very small pits. Fig. 7 plots the effect of pit-depth tolerance $\delta_{\mathrm{p}}$ and spanning length $L$ on the total pit area, for a sample taken after an intermediate pass. For very small $\delta_{\mathrm{p}}$, a large number of very minor dimples on the surface are identified as pits. At the other extreme, with large $\delta_{\mathrm{p}}$ the analysis is insufficiently sensitive to pick up any of the features of importance. As discussed in Section 6.1, at an intermediate value of $\delta_{\mathrm{p}}=0.66$ most of the relevant features are identi-

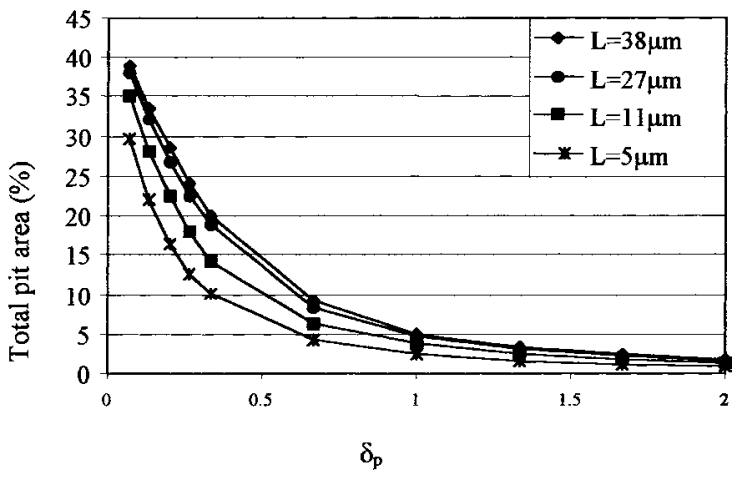

Fig. 7. Effect of changes in the pit depth tolerance $\delta_{\mathrm{p}}$ and the pit spanning length $L$ on the estimate of total pit area for an intermediate pass.

fied. At a smaller value of $\delta_{\mathrm{p}}=0.33$, some further small pits are identified, although some of these may be artefacts of the method. The value of pit area is sensitive to the exact choice of $\delta_{\mathrm{p}}$, with the correct choice depending on what size and depth of pits are considered of relevance. Fig. 7 shows that, as long as $L$ is greater than typical pit dimensions (e.g. $L \geq 22 \mu \mathrm{m}$ ), the analysis is not sensitive to this parameter.

\subsubsection{Roll marks}

A similar sensitivity analysis was performed for identification of roll marks. The parameters used in this algorithm are the roll-mark depth and slope tolerances $\delta_{\mathrm{m}}$ and $\phi$ and the roll-mark spanning length $L$. Similar results to those shown in Fig. 7 demonstrated that, as long as $L$ is taken to be wider than the roll marks, results are insensitive to the choice of this parameter. For the surfaces studied here, $L$ should be $>16 \mu \mathrm{m}$. The relative contributions of the height and slope criteria (Eq. (4)) to the roll-mark area, and the sensitivity to the choice of the depth tolerance $\delta_{\mathrm{p}}$ are given in Fig. 8. This figure shows that the roll-mark area is sensitive to the choice of depth tolerances $\delta_{\mathrm{m}}$. For small values of $\delta_{\mathrm{m}}$, the roll-mark area increases significantly as virtually all the features on the strip surface are sufficiently deep, high or steep to 'count' as roll marks. Fig. 8 shows that, for a value of slope tolerance $\phi=10$, the contribution from the slope criterion is small. As for the pit identification, the choice of $\delta_{\mathrm{m}}$

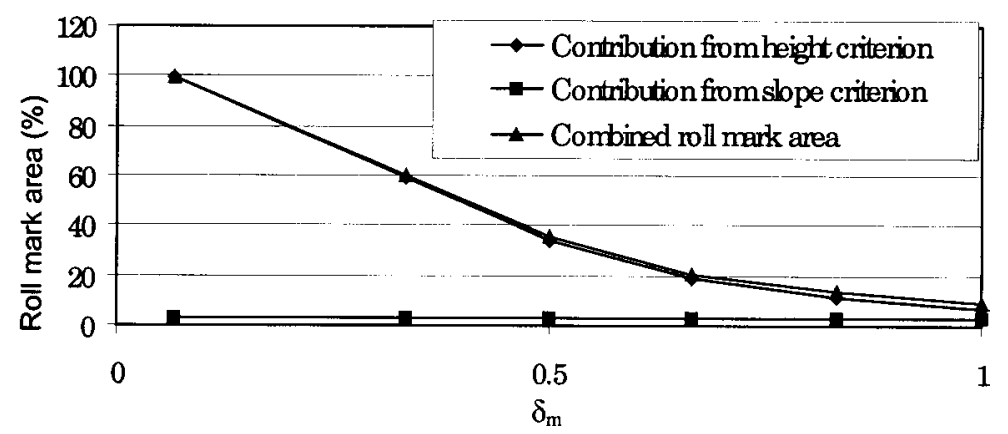

Fig. 8. Effect of changes in the roll-mark depth tolerance $\delta_{\mathrm{m}}$ and the relative contributions of the depth and slope criteria on the estimate of roll-mark area for an intermediate pass $(\phi=10, L=27 \mu \mathrm{m}$ and $M=11 \mu \mathrm{m})$. 


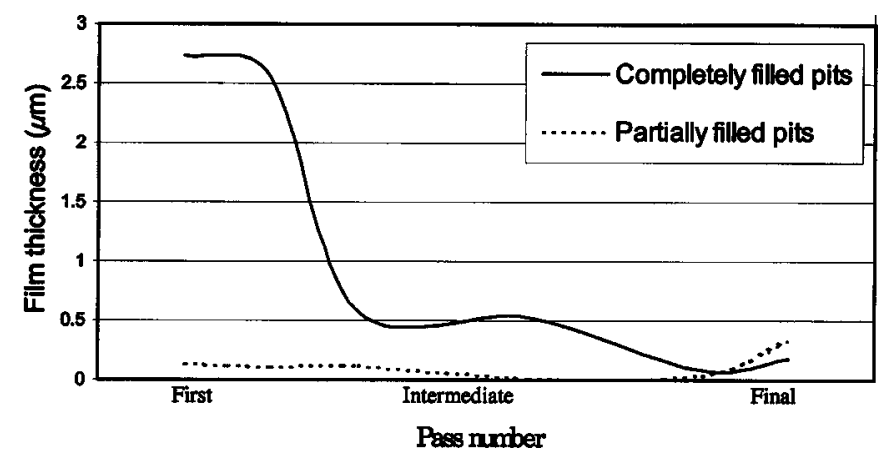

Fig. 9. Oil film thickness due to trapped oil estimated by changes in the pit volume during rolling.

should be made based on the application. It is believed that only large or steep roll marks will affect the visual appearance, so that relatively large values of the depth and slope tolerances were chosen in the illustrative examples, Fig. $3 f$ and Fig. 5.

Section 5.2 explains how the length $M$ is used to verify the features that extend significantly in the rolling direction. For the surfaces studied, a value of $M=11 \mu \mathrm{m}$ was found to achieve the required effect. Results did not change significantly when $M$ was increased to $32 \mu \mathrm{m}$. The lower value is to be preferred, as this reduces the accuracy needed in aligning the samples before making interferometry measurements.

\section{Estimate of lubrication conditions}

As the samples were collected from positions on the coil with reduced rolling speeds, hydrodynamic lubrication effects due to the entraining action at normal rolling speeds will not be present. However, the amount of oil trapped in pits may still be representative of that at higher speeds. Hence, it is instructive to make some estimate, from the change in strip surface during rolling, of the way in which the trapped oil might be expelled from the pits and so contribute to the lubrication regime. Firstly, the methodology described in Section 5.1 is used to identify the location and depth of the pits for samples from each pass, using the parameters $\delta_{\mathrm{p}}=0.33$ and $L=27 \mu \mathrm{m}$. It is unclear to what extent the oil can fill the pits at the entry to the bite, so that two alternatives were considered, either that the lubricant fills all the valleys or that pits are only filled up to a depth of $0.5 \mu \mathrm{m}$. This latter assumption is based on the supposition that there may be insufficient time at the inlet for oil to fill up the deeper pits. The volume of oil trapped in the pits before and after each pass can then be estimated. The difference is oil which is expelled from the pits into the surrounding area during the pass. By supposing that this expelled oil spreads out over an area around the pit equal to the area of the pit itself, we can make an approximate estimate of the film thickness around the pits at the exit of the bite. Estimates of the film thickness due to expelled lubricant are shown in Fig. 9, for the assumptions of either completely filled pits or pits which are partially filled

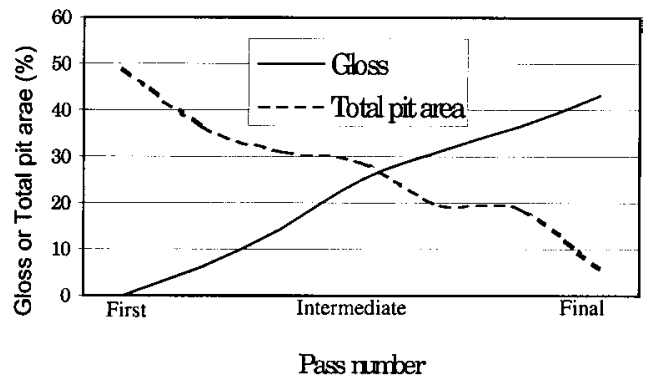

Fig. 10. Correlation between the change in total pit area and surface gloss during the pass schedule.

to a depth of $0.5 \mu \mathrm{m}$. Although this estimate of film thickness is very approximate, Fig. 9 indicates that there may be a substantial film of oil caused by this expelled lubricant, perhaps of the order of $0.1 \mu \mathrm{m}$. By comparison, hydrodynamic entraining action at normal rolling speeds is only expected to generate films of the order of $0.01 \mu \mathrm{m}$ in thickness.

\section{Effect of remnant features on sheet quality}

Current industrial practice is to quantify sheet quality by measuring the surface reflectance. The gloss, defined as the percentage of incident light reflected back, was measured on the strip samples described in Section 2, at an incident angle of $20^{\circ}$, using a standard reflectance measurement device. Typical results are shown in Fig. 10, showing a gradual improvement in gloss with pass number. Also shown on Fig. 10 is the change in total pit area during the pass schedule, estimated using a value of $\delta_{\mathrm{p}}=0.33$, showing how the increase in gloss correlates well with the reduction in pit area. This result demonstrates that a good understanding of the evolution of surface features is a key to modelling the industrially practical changes in surface reflectance.

\section{Conclusions}

A novel method has been described to identify surface features on cold-rolled stainless steel strip from three 
dimensional profilometry data. The aim of the approach is to detect and quantify automatically those features that can be observed in optical or SEM micrographs, to give quicker, more objective and more accurate results than visual methods. Algorithms for identifying pits and roll marks are developed, based on observations of these features on the strip surface. Results presented in Section 6 show that these algorithms are effective in identifying pits and roll marks for samples taken after an intermediate and the final passes of rolling. By suitable adjustment of the tolerances used in the analysis, the method can be tailored to detect less severe features, although there may be a loss of accuracy. The choice of tolerance will depend on the perceived importance of the size and depth of the features, as discussed in Section 6.3. It is expected that application of the method, either for research or quality control purposes, would require calibration against existing surface inspection methods, taking into account the surface requirements of the product.

The methodology is applied in Section 6.2 to a series of samples taken through a pass schedule. Results show how the initially large area of deep pits (i.e. $>0.5 \mu \mathrm{m}$ in depth) is rapidly eliminated and transformed into shallow pits. These shallower pits are not eliminated effectively until the final passes where a smoother roll is used. Similarly, the roll mark area only falls significantly with use of the smoother roll. The pit identification method is used to estimate the effect of trapped oil on lubrication. By following the change in pit volume during the pass schedule it is possible to estimate how much oil is expelled from the pits during each pass. Results suggest that this expelled oil will contribute significantly to lubrication in the roll bite. A good correlation between strip surface reflectance and pit area is observed, illustrating that a good understanding of the evolution of surface features is a key to modelling the industrially practical changes in surface reflectance.

The method described in this paper gives similar results to the Fourier transform filtering method developed recently by Le and Sutcliffe [17] to identify pits on rolled aluminium strip. The simplicity in implementing that method makes it an attractive alternative to the work presented in this paper. The advantage of the current work is that it is easy to apply a variety of criteria tailored to the characteristics of the different features observed on the strip. The wavelet analysis method described by Jiang et al. [18], although more complicated to implement, is also an useful alternative, as it can deal efficiently with features occurring at different length scales.

\section{Acknowledgements}

The authors are most grateful for permission to use the samples and test results described in Sections 2 and 8, which were collected by staff at Avesta Sheffield and Corus plc. The technical assistance of staff at these collaborating compa- nies, including Dr Didier Farrugia and Mr Peter Freeman, at Corus, Swinden Technology Centre, and Messrs. Ken King and Andrew Backhouse at Avesta Sheffield, is gratefully acknowledged, as is the financial support of the collaborating companies, the Engineering and Physical Sciences Research Council and the Isaac Newton Trust. The authors are grateful for additional measurements and analysis by Mr Fotios Georgiades.

\section{References}

[1] W.R.D. Wilson, S. Sheu, Real area of contact and boundary friction in metal forming, Int. J. Mech. Sci. 30 (1988) 475-489.

[2] M.P.F. Sutcliffe, Surface asperity deformation in metal forming processes, Int. J. Mech. Sci. 30 (1988) 847-868.

[3] W.R.D. Wilson, J.A. Walowit, 1972. An isothermal hydrodynamic lubrication theory for strip with front and back tension, Tribology Convention, Institute of Mechanical Engineers, London, pp. 164-172.

[4] M.P.F. Sutcliffe, K.L. Johnson, Experimental measurements of lubricant film thickness in cold strip rolling, Proc. Inst. Mech. Eng. 204 (1990) 263-273.

[5] S. Sheu, W.R.D. Wilson, Mixed lubrication of strip rolling, STLE, Tribol. Trans. 37 (1994) 483-493.

[6] T. Mizuno, M. Okamoto, Effects of lubricant viscosity at pressure and sliding velocity on lubricating conditions in the compression friction test on sheet metals, J. Lubric. Techn. 104 (1982) 53-59.

[7] J. Kihara, S. Kataoka, T. Aizawa, Quantitative evaluation of micro-pool lubrication mechanism, J. Jpn. Soc. Techn. Plast. 33-376 (1992) 556-561.

[8] H.S. Lin, N. Marsault, W.R.D. Wilson, A mixed lubrication model for cold strip rolling. Part I. Theoretical, Tribol. Trans. 41 (1998) 317-326.

[9] F. Fudanoki, 1997. Development and evaluation of model for mechanism of formation of surface properties of cold-rolled stainless steel, First International Conference on Tribology in Manufacturing Processes, Gifu, Japan, pp. 378-383.

[10] Z. Wang, K. Dohda, N. Yokoi, Y. Haruyama, 1997, Outflow behaviour of lubricant in micro-pits in metal forming, First International Conference on Tribology in Manufacturing Processes, Gifu, Japan, pp. $77-82$.

[11] Z. Wang, K. Kondo, T. Mori, A consideration of optimum conditions for surface smoothing based on lubricating mechanisms in ironing process, J. Eng. Ind. 117 (1995) 351-356.

[12] S. Lo, W.R.D. Wilson, 1997. A theoretical model of micro-pool lubrication in metal forming, First International Conference on Tribology in Manufacturing Processes, Gifu, Japan, pp. 83-90.

[13] L. Gjønnes, Quantitative characterisation of the surface topography of rolled sheets by laser scanning microscopy and Fourier transformation, Metall. Mater. Trans. A 27A (1996) 2338-2346.

[14] T. Ohkubo, J. Shibata, K. Sato, K. Seki, Y. Odaka, 1997, Quantitative analysis for surface topography of aluminium foil by SEM and AFM, First International Conference on Tribology in Manufacturing Processes, Gifu, Japan, pp. 59-64.

[15] L.A. Kuznetsov, The models of a strip surface roughness formation during cold rolling, Model. Measure. Contr. B 57 (3) (1995) 50-64.

[16] D. Schmoeckel, J. Staeves, J., 1997. Function-orientated 3D filtering for tribological assessment of sheet metal surfaces in deep-drawing, 7th International Conference on Metrology and Properties of Engineering Surface, pp. 438-444.

[17] H.R. Le, M.P.F. Sutcliffe, Analysis of surface roughness of cold rolled aluminium foil, Wear 244 (2000) 72-79.

[18] X.Q. Jiang, L. Blunt, K.J. Stout, Proceedings of the Institution of Mechanical Engineers, Part H, J. Eng. in Med. 213, (1999) 49-68. 